\title{
Relationship between migraine and right-to-left shunt in children: editorial
}

\author{
Werner Budts
}

Received: 12 August 2010 /Accepted: 15 September 2010/Published online: 28 September 2010

(C) Springer-Verlag 2010

Migraine is a common type of headache and is considered to be one of the most important disabling disorders worldwide. The prevalence of migraine increases with age until it reaches the peak prevalence of $27 \%$ in women and $8 \%$ in men in the fourth decade of life [11]. Migraine is a complex disorder in which genetic, environmental, behavioral, and other unidentified factors interact to trigger typical migraine attacks. In the late 1990s, it was suggested for the first time that migraines, especially those with aura, are associated with the presence of a right-to-left shunt (prevalence of $23 \%$ for migraine without aura, $48 \%$ for migraine with aura) [1]. Moreover, in patients with a rightto-left shunt, migraine was more prevalent $(48 \%$ for migraine with aura) than in those without a shunt [14]. These observations were independent from the structural anomalies causing the right-to-left shunt: patent foramen ovale, atrial septal defect, or pulmonary arteriovenous malformation.

In this issue, Sarisoy et al. [10] reported similar findings: $65 \%$ of the children with migraine with aura had a documented right-to-left shunt, which was significantly higher than the prevalence of a right-to-left shunt in patients with migraine without aura or with no migraine $(25 \%$ and $20 \%$, respectively). These authors are the first to show an association between migraine with aura and right-to-left shunt in a pediatric population (mean age 11 years).

A few hypotheses were proposed to explain the potential relationship between a right-to-left shunt and migraine. First, vasoactive trigger substances of the venous circula-

\footnotetext{
W. Budts $(\square)$

Congenital and Structural Cardiology,

University Hospitals Leuven,

Herestraat 49,

3000 Leuven, Belgium

e-mail: werner.budts@uz.kuleuven.ac.be
}

tion may enter through a right-to-left shunt into the systemic circulation, inducing cerebral vascular instability, thereby provoking a typical migraine attack. It is suggested that these trigger substances, such as serotonin and/or (micro)thrombi, are normally neutralized in the lung filter. However, a right-to-left shunt may bypass this neutralization process so that these non-deactivated substances enter the systemic circulation [1]. This hypothesis fits with the increased risk for stroke or transient ischemic attacks in patients with migraine, especially in those with aura. Second, a particular genetic substrate may determine migraine. Migraines seem to be more prevalent in patients with congenital heart defects or connective tissue diseases, even independent from the existence of a right-to-left shunt $[3,13]$. In the presence of a right-to-left shunt, migraine occurs even more frequently. All these hypotheses are referring to adolescents and adults, but as suggested by Sarisoy et al., it may also be applied to children.

In addition that migraine (with aura) seems to be associated with a higher prevalence of a right-to-left shunt, some people believe that migraine occurs only in patients with at least moderate to severe shunts [8]. Observational studies are ongoing to evaluate whether specific characteristics of patent foramen ovale, such as the presence of an atrial septal aneurysm, a floppy inter-atrial septum, or long tunnel between septum primum and septum secundum, determine the occurrence or influence the severity, the frequency, or the duration of migraine attacks. Sarisoy et al. found no influence of the shunt on the clinical features of migraine. Because of the high variability of migraine attacks, it is possible that most observational studies are underpowered to show differences in the clinical presentation of migraine. Analyses of larger series are ongoing.

Finally, the potential relationship between a right-to-left shunt and migraine was enforced by retrospective non- 
randomized trials which showed that the prevalence of migraine, and especially with aura, decreased significantly after percutaneous per-catheter shunt closure. Prevalence of migraine after patent foramen ovale closure was reduced by $59 \%$ and migraine with aura by $74 \%$. Most of these patients suffered earlier from a stroke or transient ischemic attack due to a paradoxical embolism through the patent foramen ovale, which was the indication for shunt closure. The same decrease in prevalence was found in patients with embolized pulmonary arteriovenous malformation, from $45 \%$ before to $35 \%$ after for migraine and from $33 \%$ before to $19 \%$ after for migraine with aura. Results were similar in patients with percutaneous atrial septal defect closure [7]. These studies were criticized because of the retrospective design and the lack of control groups. Indeed, it is possible that "the procedure of closing a shunt" induces a placebo effect and that the decreased prevalence is only "placeborelated." However, most migraine studies suggest that a placebo effect is limited to a reduction of $20 \%$ to a maximum of $40 \%$, whereas in the retrospective studies, the reduction was up to $70 \%$. Others believe that the improvement of migraine is not related to shunt closure but to the concomitant use of antiplatelets or oral anticoagulants. Indeed, all patients with a closed patent foramen ovale or atrial septal defect were treated with antiplatelets or oral anticoagulants so that these drugs may be responsible for the changes in migraine. But the effect of shunt closure on migraine seems to persist over the years, even after the discontinuation of these drugs. Moreover, after the closure of a pulmonary arteriovenous malformation, even if antiplatelets or oral anticoagulants are not administered, the effect on migraine is still noticed. To overcome the problems related to retrospective study design, several centers have started to follow their patients prospectively after shunt closure (Table 1 ). Similar results as in the retrospective studies were obtained: at least a $50 \%$ decrease in migraine prevalence or a similar decrease in severity of migraine attacks [12]. These findings supported the hypothesis that a right-to-left shunt may play a pathophysiological role in some types of the migraine.
But, before one believes in this relationship, prospective randomized trials are needed to answer the following crucial questions: (1) Is there indeed a relationship between migraine and a right-to-left shunt and (2) does shunt closure influence migraine attacks? With these questions in mind, the MIST I trial was performed; patients with migraine with aura were randomized for a percutaneous patent foramen ovale closure or a sham procedure [2]. The higher prevalence of patent foramen ovale and right-to-left shunt in patients with migraine with aura was confirmed, but the trial failed to show a difference in the cessation of migraine between the two groups of patients. In exploratory analysis, excluding two outliers, the implant group demonstrated a greater reduction in migraine-related headache days, which suggests the influence of closure on the number of migraine attacks. These results and the potential risk for underpowering terminated prematurely another earlier organized, randomized controlled migraine trial (FORMAT) [9]. The primary endpoint of this study was the change in frequency of migraine with aura attacks. The FORMAT dataset showed that the number of migraine with aura days was reduced to at least $50 \%$ after 6 months, in both groups: one only treated with aspirin and the other treated with percutaneous patent foramen ovale closure and aspirin. Again, these data suggest that migraine may be influenced by thromboembolic prevention where aspirin and/or patent foramen ovale closure inhibit paradoxical microembolism and aspirin alone prevents systemic microthromboemboli. The article by Sarisoy et al. motivates investigators to observe whether shunt closure in children would also influence migraine patterns.

However, today, we are far from the conclusion that right-to-left shunt closure is indicated in patients with migraine (with aura). Because of the consistent findings, a right-to-left shunt might play a role in the pathophysiological process of some types of migraine attacks, but the primum movens has still to be determined. Indeed, as a final thought, there are still a substantial number of patients with migraine with no right-to-left shunt to whom these hypotheses do not apply.

Table 1 Changes in the prevalence of migraine in prospective patent foramen ovale and atrial septal defect trials

\begin{tabular}{lcccccc}
\hline & Year & Type & $n$ & FU (months) & Prevalence M pre (\%) & Prevalence M post (\%) \\
\hline Luermans et al. [4] & 2008 & PFO & 92 & 6 & 29 & $11^{\mathrm{a}}$ \\
Vigna et al. [12] & 2009 & PFO & 53 & 6 & 100 & $66^{\mathrm{a}}$ \\
Papa et al. [6] & 2009 & PFO & 75 & 12 & 100 & $53^{\mathrm{a}}$ \\
Luermans et al. [5] & 2009 & ASD & 70 & 12 & 34 & $12^{\mathrm{a}}$
\end{tabular}

$P F O$ patent foramen ovale, $A S D$ atrial septal defect, $n$ number, $F U$ follow-up time, $M$ pre migraine pre-shunt closure, $M$ post migraine after shunt closure

${ }^{\mathrm{a}}$ Significant 


\section{References}

1. Anzola GP, Magoni M, Guindani M et al (1999) Potential source of cerebral embolism in migraine with aura: a transcranial Doppler study. Neurology 52(8):1622-1625

2. Dowson A, Mullen MJ, Peatfield R et al (2008) Migraine Intervention With STARFlex Technology (MIST) trial: a prospective, multicenter, double-blind, sham-controlled trial to evaluate the effectiveness of patent foramen ovale closure with STARFlex septal repair implant to resolve refractory migraine headache. Circulation 117(11):1397-1404

3. Hermans H, Post MC, Thijs V et al (2007) Increased prevalence of migraine in adult congenital heart disease. Heart 93(3):361-362

4. Luermans JG, Post MC, Temmerman F et al (2008) Closure of a patent foramen ovale is associated with a decrease in prevalence of migraine: a prospective observational study. Acta Cardiol 63 (5):571-577

5. Luermans JG, Post MC, Temmerman F et al (2009) Is a predominant left-to-right shunt associated with migraine? A prospective atrial septal defect closure study. Catheter Cardiovasc Interv 74(7):1078-1084

6. Papa M, Gaspardone A, Fragasso G et al (2009) Usefulness of transcatheter patent foramen ovale closure in migraineurs with moderate to large right-to-left shunt and instrumental evidence of cerebrovascular damage. Am J Cardiol 104 (3):434-439

7. Post MC, Budts W (2006) The relationship between migraine and right-to-left shunt: fact or fiction? Chest 130(3):896-901

8. Rigatelli G, Dell'avvocata F, Cardaioli P et al (2008) Migrainepatent foramen ovale connection: role of prominent eustachian valve and large Chiari network in migrainous patients. Am J Med Sci 336(6):458-461

9. Sarens T, Herroelen L, Van Deyk K, Budts W (2009) Patent foramen ovale closure and migraine: are we following the wrong pathway? J Neurol 256(1):143-144

10. Sarisoy S, Aydin OF, Sungur M et al. (2010) The relationship between migraine and right-to-left shunt in children. Eur J Pediatr 431(3):365-370. doi:10.1007/s00431-010-1303-7

11. Stewart WF, Shechter A, Rasmussen BK (1994) Migraine prevalence. A review of population based studies. Neurology 44 (6 Suppl 4):S17-S23

12. Vigna C, Marchese N, Inchingolo V et al (2009) Improvement of migraine after patent foramen ovale percutaneous closure in patients with subclinical brain lesions: a case-control study. JACC Cardiovasc Interv 2(2):107-113

13. Vis JC, Timmermans J, Post MC et al (2009) Increased prevalence of migraine in Marfan syndrome. Int J Cardiol 136(3):330-334

14. Wilmshurst P, Nightingale S (2001) Relationship between migraine and cardiac and pulmonary right-to-left shunts. Clin Sci (Lond) 100(2):215-220 